\title{
Metabolomic and elemental profiling of human tissue in kidney cancer
}

\author{
Joanna Nizioł ${ }^{1}$ (C) . Valérie Copié ${ }^{2} \cdot$ Brian P. Tripet $^{2} \cdot$ Leonardo B. Nogueira $^{3} \cdot$ Katiane O. P. C. Nogueira $^{4}$. \\ Krzysztof Ossoliński $^{5} \cdot$ Adrian Arendowski $^{1} \cdot$ Tomasz Ruman $^{1}$
}

Received: 8 November 2020 / Accepted: 22 February 2021 / Published online: 4 March 2021

(c) The Author(s) 2021

\begin{abstract}
Introduction Kidney cancer is one of the most frequently diagnosed and the most lethal urinary cancer. Despite advances in treatment, no specific biomarker is currently in use to guide therapeutic interventions.

Objectives Major aim of this work was to perform metabolomic and elemental profiling of human kidney cancer and normal tissue and to evaluate cancer biomarkers.

Methods Metabolic and elemental profiling of tumor and adjacent normal human kidney tissue from 50 patients with kidney cancer was undertaken using three different analytical methods.

Results Five potential tissue biomarkers of kidney cancer were identified and quantified using with high-resolution nuclear magnetic resonance spectroscopy. The contents of selected chemical elements in tissues was analyzed using inductively coupled plasma optical emission spectrometry. Eleven mass spectral features differentiating between kidney cancer and normal tissues were detected using silver-109 nanoparticle enhanced steel target laser desorption/ionization mass spectrometry. Conclusions Our results, derived from the combination of ICP-OES, LDI MS and 1H NMR methods, suggest that tissue biomarkers identified herein appeared to have great potential for use in clinical prognosis and/or diagnosis of kidney cancer.
\end{abstract}

Keywords Human kidney tissue $\cdot$ Cancer $\cdot$ Biomarkers $\cdot$ Metallomics $\cdot$ Metabolomics

\section{Introduction}

Kidney cancer is one of the most frequently diagnosed metabolic diseases of the urinary tract. More than 400 thousand new cases of kidney cancer and nearly 180 thousand deaths occurred in 2018 (Bray et al., 2018). Based on histological classification, a number of different types of kidney cancers were classified including both benign tumors like adenoma,

Joanna Nizioł

jniziol@prz.edu.pl

1 Faculty of Chemistry, Rzeszów University of Technology, 6 Powstańców Warszawy Ave., 35-959 Rzeszów, Poland

2 The Department of Chemistry and Biochemistry, Montana State University, Bozeman, MT 59717, USA

3 Department of Geology, Federal University of Ouro Preto, Ouro Preto, Minas Gerais, Brazil

4 Department of Biological Sciences, Federal University of Ouro Preto, Ouro Preto, Minas Gerais, Brazil

5 Department of Urology, John Paul II Hospital, Grunwaldzka 4 St., 36-100 Kolbuszowa, Poland oncocytoma and angiomyolipoma (AML) and the most common malignant type of kidney cancer, renal cell carcinoma (RCC). RCC is accounting for approximately ninety percent of all neoplasms originating from the kidney. There are three main types of RCC known, namely clear cell RCC (ccRCC), papillary RCC (pRCC) and chromophobe RCC (cRCC). Mentioned types may differ in stage, grade, and cancer-specific survival. Other subtypes of RCC are very rare and include angiomyolipoma (AML), collecting duct carcinoma (CDC), or simple renal cyst (SRC) (Hsieh et al., 2017).

Currently, RCC diagnosis is based on magnetic resonance imaging, ultrasound examination or computed tomography. Unfortunately, more than $60 \%$ of RCC cases are diagnosed incidentally. This tumor is difficult to detect, especially in its early stages, due to the lack of characteristic symptoms including lack of the classic triad of visible haematuria, flank pain and palpable abdominal mass symptoms. Poor prognosis and high mortality rate are related to metastases and resistance to chemotherapy and radiotherapy. The 5 -year survival rates of patients with metastatic disease are 
less than ten percent (Lim et al., 2013). In spite of great efforts there are still no clinically available biomarkers for early detection, diagnosis or prognosis of kidney cancers. Analysis of metabolic profiles from tissues and biofluids is a promising approach for the discovery of biomarkers that would enhance our abilities to predict cancer progression and to assess the effectiveness of cancer treatment (Gupta et al., 2020).

Over the past two decades, two major analytical platforms have been employed for metabolomic analysis of kidney cancer samples of various types: (i) mass spectrometry (MS) (Lin et al., 2012) and (ii) nuclear magnetic resonance (NMR) (Gao et al., 2012). Tissues from patients with kidney cancer have been studied using primarily mass spectrometry (Gupta et al., 2020). The Catchpole et al. was one of the first to use profiling of low molecular weight compounds, such as sugars, lipids and amino acids to characterize the metabolic signature of RCC (Catchpole et al., 2011). Later, various groups performed focused profiling studies based mainly on LC- and GC-MS approaches (Shim et al., 2014; Wettersten et al., 2015). Interestingly, there exist no published studies of laser mass spectrometry data of human tissue extracts to date.

Nuclear magnetic resonance-based metabolomic studies of RCC tissue samples are relatively rarely found in literature (Gao et al., 2008). Previously, ${ }^{1} \mathrm{H}$ magic angle spinning (MAS) NMR was used to analyze kidney carcinoma biopsy samples (Moka et al., 1998; Tate et al., 2000). To our knowledge, there is only one publication concerning the utilization of ${ }^{1} \mathrm{H}$ NMR in the metabolomic analysis of human RCC tissues-in 2012 Gao et al. applied ${ }^{1} \mathrm{H}$ NMR to the characterization of RCC metastases in tissue extracts (Gao et al., 2012). No published studies that combine both NMR, LDI MS obtained from tissues of patients with kidney cancer have been reported to date.

Many studies have suggested a relationship between toxic metals and trace elements (TMTE) and the development of carcinoma in humans (Mulware, 2013). Toxic elements can cause genetic and epigenetic effects that may result in increased risk of different cancer types (Mishra et al., 2014). ICP-OES is one the most frequently used approaches to determine metal elements and speciation in biological samples, and has been employed to assess the effects of TMTE on kidney tissue samples from patients with RCC (AbdelGawad et al., 2020). The results showed that the differences in the concentration of TMTE between normal and malignant tissue could be used as a biomarker of this disease.

In this study, we performed the first targeted and untargeted metabolomic and metallomic profiling of 100 human tissue samples using three different analytical platforms: NMR, ICP-OES and LDI MS. The value of laser desorption/ionization MS using a 109-silver nanoparticleenhanced steel target $\left({ }^{109} \mathrm{AgNPET}\right.$ LDI MS) approach for metabolomics has been demonstrated in the detection of metabolites in plant and human tissues (Arendowski et al., 2018; Nizioł et al., 2019, Nizioł, Ossoliński, et al., 2020, 2021; Nizioł, Sunner, et al., 2020).

\section{Materials and methods}

\subsection{Materials and equipment}

${ }^{109} \mathrm{AgNPET}$ was prepared as described previously (Nizioł et al., 2013). 2,5-Dihydroxybenzoic acid (DHB) was purchased from Aldrich. Silver-109 was purchased from BuyIsotope (Sweden). All solvents were of minimum 'HPLC purity', except for methanol and water (LCMS grade, Fluka) Deuterium oxide $\left(\mathrm{D}_{2} \mathrm{O}\right)$ and 4,4-dimethyl-4-silapentane1-sulfonic acid (DSS) NMR reagents were purchased from Sigma Inc. Nitric acid 65\% p.a EMSURE ISO and Hydrogen peroxide $30 \%$ p.a. EMSURE ACS ISO was purchased from Merck.

\subsection{Collection of tissue samples}

Tissue samples were collected from 50 kidney cancer patients (20 females, 30 males, average age 69) undergoing surgical treatment, following detailed clinical questioning at John Paul II Hospital in Kolbuszowa (Poland). The study protocol was approved by local Bioethics Committee at the University of Rzeszow (Poland, permission no. 2018/04/10). All the patients in this study were of Caucasian race. All research was performed in accordance with relevant guidelines and regulations. Specimens and clinical data from patients involved in the study were collected with informed consent. All laboratory test results (complete blood count, urine analysis, bleeding profile, kidney function tests, CRP) were within normal ranges. Each patient donated $1 \mathrm{~cm}^{3}$ of renal cancer tissue ('cancer') dissected together with a small fragment of normal tissue $\left(<1 \mathrm{~cm}^{3}\right.$, 'controls') removed ex vivo during radical nephrectomy. Samples were immediately frozen and stored at $-60{ }^{\circ} \mathrm{C}$ until further use. The pathological and clinical characteristics of the patients are presented in supplementary material table (Table S1).

\subsection{Preparation of tissue extracts for NMR and LDI MS studies}

A weighted amount (10-168 mg) of sectioned tissue was transferred to a $2 \mathrm{ml}$ centrifuge tube, then $900 \mu \mathrm{l}$ of a 1:2 $\mathrm{MeOH} / \mathrm{CHCl}_{3}(1: 2$, v/v) solution was added and homogenized with glass beads for $45 \mathrm{~s}$. Next, a volume of $120 \mu \mathrm{l}$ of cold $\mathrm{H}_{2} \mathrm{O}$ was added to each tube and then again homogenized two times for $45 \mathrm{~s}$ with a 5 -min break time. The samples were placed at $-20{ }^{\circ} \mathrm{C}$ for $1 \mathrm{~h}$ and then centrifuged at 
$14,000 \times g$ for $10 \mathrm{~min}$ at $4{ }^{\circ} \mathrm{C}$ to remove cells and other precipitated material. The polar (upper) phase was transferred to new $1.5 \mathrm{ml}$ microcentrifuge tube, similarly non-polar (lower) phase was transferred to new $1.5 \mathrm{ml}$ microcentrifuge tube. Finally, from all resulting samples, $50 \mu \mathrm{l}$ volumes were taken and used for ${ }^{109}$ AgNPET LDI MS analyses. The rest of the sample was lyophilized to complete dryness using a SpeedVac vacuum concentrator ( $c a .1$ mbar), with no heat. Dried material was re-suspended in $600 \mu \mathrm{L}$ of NMR buffer consisting of $25 \mathrm{mM} \mathrm{NaH} \mathrm{PO}_{4} / \mathrm{Na}_{2} \mathrm{HPO}_{4}, 0.4 \mathrm{mM}$ imidazole, $0.25 \mathrm{mM}$ 4,4-dimethyl-4-silapentane-1-sulfonic acid (DSS) in $90 \% \mathrm{H}_{2} \mathrm{O} / 10 \% \mathrm{D}_{2} \mathrm{O}, \mathrm{pH} 7.0$. Following re-suspension, samples were centrifuged at $21,000 \mathrm{rpm}$ for $1 \mathrm{~min}$ to pellet insoluble debris, and then transferred to $5 \mathrm{~mm} \mathrm{NMR}$ tubes for NMR metabolomics analysis.

\subsection{NMR spectra acquisition and data processing}

NMR spectra acquisition, preprocessing and postprocessing were conducted as described recently (Nizioł, Ossoliński, et al., 2020; Nizioł, Sunner, et al., 2020). NMR data sets were normalized by tissue weight.

\subsection{MS spectra acquisition and preprocessing}

Volume of $0.3 \mu \mathrm{l}$ of each sample was placed on a ${ }^{109} \mathrm{AgNPET}$ target plate and allowed to evaporate to dryness at room temperature, then target was inserted into a MALDI-type ToF/ ToF mass spectrometer. Laser desorption ionization mass spectrometry (LDI MS) experiments were performed with a Bruker Autoflex Speed ToF mass spectrometer in positiveion reflectron mode. The apparatus was using a SmartBeam II $355 \mathrm{~nm}$ laser. Laser impulse energy was approximately $100-190 \mu \mathrm{J}$, laser repetition rate of $1000 \mathrm{~Hz}$. Deflection was set on $\mathrm{m} / \mathrm{z} 80$, measurements were made within $\mathrm{m} / \mathrm{z}$ range of 80-2000 Da. Spot of each extract was ablated by $20 \mathrm{k}$ laser shots with a default random walk applied. All spectra were calibrated with silver ions: ${ }^{109} \mathrm{Ag}^{+}$to ${ }^{109} \mathrm{Ag}_{10}{ }^{+}$. The first ion source voltage was held at $19 \mathrm{kV}$, and the second $16.7 \mathrm{kV}$. Reflector voltages were $21 \mathrm{kV}$ (first) and $9.55 \mathrm{kV}$ (second). MS data sets were normalized by tissue weight. FlexAnalysis 4.0 program was used for data processing and analysis.

\subsection{ICP-OES analysis}

In order to determine the concentrations of major elements (Ca (calcium), Fe (iron), K (potassium), $\mathrm{Na}$ (sodium), $\mathrm{Mg}$ (magnesium)), minor elements (Mn (manganese), P (phosphorus), $\mathrm{S}$ (sulfur)) and trace elements (Cu (copper), and $\mathrm{Zn}$ (zinc)) in kidney cancer and normal tissue, 58 samples were taken for analysis with ICP-OES. All of the 58 samples were separated into three different groups according to similar mass. An analytical balance was used to verify sample mass.
A $5 \mathrm{ml}$ final solution volume was collected for all samples except for ten samples which had the highest mass; these resulted in a volume of $10 \mathrm{ml}$. The samples were weighted and placed in Teflon tubes (Savillex). $4 \mathrm{ml}$ of $\mathrm{HNO}_{3}$ and $1 \mathrm{~mL}$ of $\mathrm{H}_{2} \mathrm{O}_{2}$ were added to the highest mass samples, while $3 \mathrm{ml}$ of $\mathrm{HNO}_{3}$ and $0.5 \mathrm{ml}$ of $\mathrm{H}_{2} \mathrm{O}_{2}$ were added to all other samples. The teflon tubes were closed and placed on the heating plate at $100{ }^{\circ} \mathrm{C}$ for $24 \mathrm{~h}$. Following $24 \mathrm{~h}$ of digestion, ultrapure water was added to achieve the stated final volume of $5 \mathrm{ml}$. All Teflon tubes were weighted using an analytical balance and masses recorded. The final solutions were transferred to labeled flasks that had been washed with ultrapure water. The international standard NIST $1577 \mathrm{c}$ (bovine liver-NIST) was used between samples to monitor the instrumental performance and for quality control. The major, minor and trace elements were analyzed using the Agilent 725 Inductively Coupled Plasma Optical Emission Spectrometer. ICP-OES parameters and associated analytical conditions are reported in Table S2 (Supplementary information).

\subsection{Multivariate statistical analysis}

Metabolite data was analyzed with the use of MetaboAnalyst software 4.0 (Chong et al., 2018). Data was log-transformed and auto-scaled prior to statistical analysis. Firstly, a total of $99{ }^{1} \mathrm{H}$ NMR spectra were recorded on metabolite mixtures extracted from tissue samples of kidney cancer patients (49 tumor tissues and 50 adjacent normal tissues), 100 LDI MS spectra were recorded for the same 50 patients and 50 ICPOES spectra were recorded for 58 kidney cancer patients (28 tumor tissues and 28 adjacent normal tissues). Resulting metabolite profiles from NMR, and MS and ICP-OES data sets were then subjected to unsupervised Principal Component Analysis (PCA). The separation between these two groups was examined Orthogonal Partial Least Squares Discriminant Analysis (OPLS-DA). The overall quality of the OPLS-DA models was assessed by examining the goodness of fit $\left(\mathrm{R}^{2} \mathrm{Y}\right)$ and the predictive ability of the models $\left(Q^{2}\right)$. S-plots were generated to identify metabolites whose level changes were most significantly responsible for groups separation. Metabolites with $|p|>0.05$ (magnitude) and $\mid p$ (corr) $\mid>0.5$ (reliability) were considered as potential biomarker candidates to distinguish kidney cancer tissues from normal controls. To test the accuracy of the multivariate statistical models, and to rule out that the observed separation in the OPLS-DA is due to chance $(\mathrm{p}<0.05)$, permutation tests were performed with 2000 -fold repetition. Statistical significance of metabolite level differences was assessed with paired parametric t-test with Mann-Witney and Bonferroni correction. P-values and false discovery rates (FDR; q-value) less than 0.05 were considered statistically significant. In addition, receiver operating curve (ROC) analyses 
with random forest algorithm were undertaken to evaluate the diagnostic value of all and selected metabolites, elements and mass features for all models. Chemometric tools such as 2D PCA and OPLS-DA were also used to assess metabolic profile similarities and differences between malignant types of kidney cancer (ccRR, chRCC, pRCC, CDC, SRC) and benign (oncocytoma and AML). The relevant metabolic changes associated with age and gender were identified by one-way analysis of variance (ANOVA). To identify the most relevant metabolic pathways involved with kidney cancer, the metabolic pathway analysis was applied with MetaboAnalyst version 5.0 based on the Kyoto Encyclopedia of Genes and Genomes (KEGG) pathway library for Homo sapiens. Data was normalized with log-transformation and autoscaling. Enrichment analysis has been computed with global test. Topology analysis was based on Relativebetweenness Centrality. Quantitative enrichment analysis was conducted based on Small Molecule Pathway Database (SMPD). Each pathway was classified according to statistical p-value, Holm p (p-value adjusted by Holm-Bonferroni method) and FDR (p-value adjusted using False Discovery Rate), calculated from pathway topology analysis.

\section{Results}

\subsection{Distinguishing between kidney cancer and control samples by ${ }^{1} \mathrm{H}$ NMR}

98 metabolite extracts from frozen kidney tissue samples (49 cancer and 49 control) were analyzed using high-resolution 1D ${ }^{1} \mathrm{H}$ NMR to identify potential discriminant biomarkers of kidney cancer. In total, 48 metabolites were identified and quantified in each tissue sample using ${ }^{1} \mathrm{H}$ NMR spectroscopy and metabolite profiling using Chenomx. Figure 1 presents a representative overlay of control and kidney cancer patient NMR spectra (blue and red traces, respectively) showing similarity and differences in the raw spectral data (plots A and B). Visual comparison of the NMR spectra revealed significant differences in individual metabolite levels between paired tissues.

Univariate and multivariate statistical analyses of metabolite levels were employed to assess the discrimination accuracy between tumor and the paired adjacent normal tissue. These analyses also enabled identification of significantly elevated levels of polar small molecules in kidney cancer (Supplementary material Table S3). Metabolite concentrations obtained by NMR following log-transformation and auto-scaling were analyzed with principal component analysis (PCA) to assess whether the patient versus control groups could be separated based on distinct metabolite profiles. Resulting 2D and 3D PCA scores plots (Fig. 2a, b, respectively) showed that the metabolite profiles of cancer

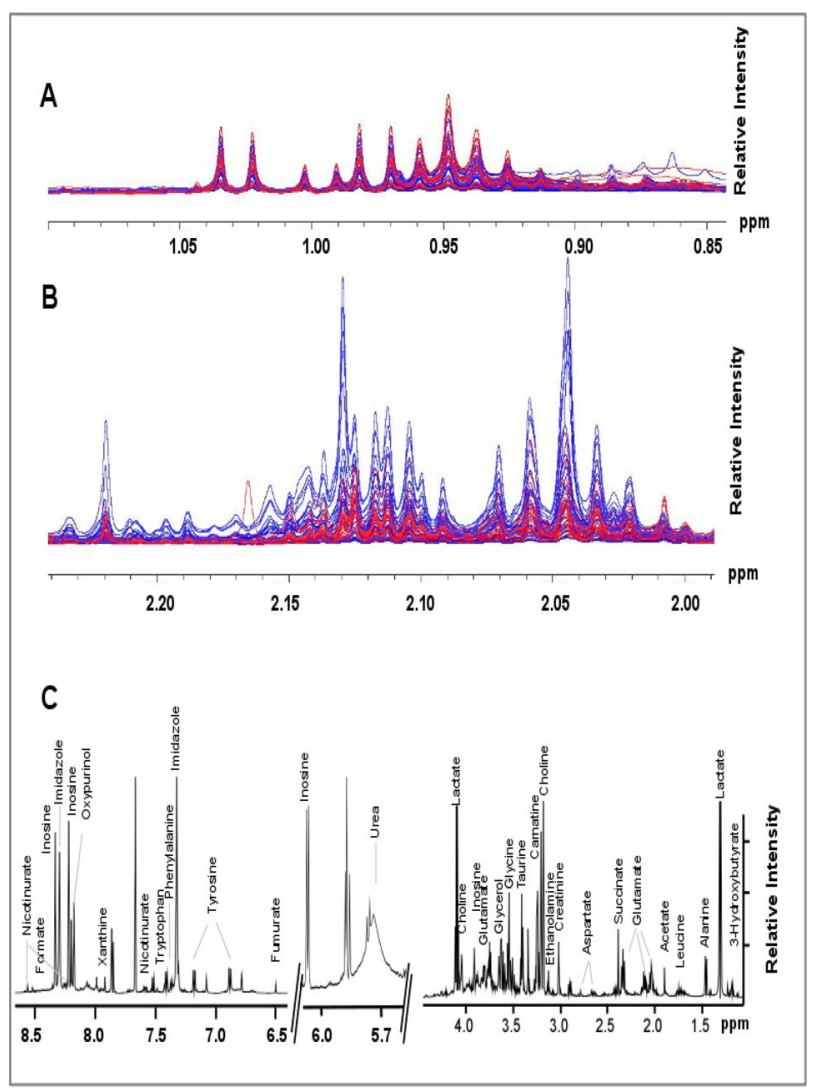

Fig. 1 Representative 1D ${ }^{1} \mathrm{H}$ NMR spectrum obtained from proteinfree, polar metabolite extracts of kidney tissue obtained from control and kidney cancer patients. a Expanded spectral region spanning the ${ }^{1} \mathrm{H}$ chemical shift range of $0.85 \mathrm{ppm}$ to $1.10 \mathrm{ppm}$, depicting the overlay of 50 NMR spectra obtained from healthy (blue traces) and cancer (red traces) kidney tissue, and illustrating no obvious spectral difference between different sample types. b Expanded spectral region spanning the ${ }^{1} \mathrm{H}$ chemical shift range of $2.00 \mathrm{ppm}$ to $2.25 \mathrm{ppm}$, depicting the overlay of 50 NMR spectra obtained from healthy (blue traces) and cancer (red traces) kidney tissue, and illustrating obvious spectral difference between different sample types. c Characteristic $1 \mathrm{D}{ }^{1} \mathrm{H}$ NMR spectrum of protein-free, polar metabolite mixtures extracted from kidney cancer tissue and spanning the ${ }^{1} \mathrm{H}$ chemical shift range of $\sim 1$ to $8.5 \mathrm{ppm}$, with characteristic signals arising from specific metabolites labeled

patients are to a large extent dissimilar from controls, with PC1 and PC2 accounting for $39.9 \%$ and $9.1 \%$ of the variance, respectively. Only few outliers were detected in the central $95 \%$ of the field of view. One pair of tissue samples identified as outliers was removed.

Group separations were also inspected using OPLS-DA which revealed a clear separation between kidney tumors and the paired adjacent tissues. The classification from OPLSDA score plot is shown in Fig. 2c. To evaluate the statistical robustness of this model 2000-permutation tests were conducted. Decent discrimination was observed between kidney cancer and normal tissues from the same patient $\left(\mathrm{Q}^{2}=0.72\right.$, $\mathrm{R}^{2} \mathrm{Y}=0.894, \mathrm{p}$-value $\left.<5 \mathrm{E}-04(0 / 2000)\right)$, revealing 
Fig. 2 Analysis of tissue metabolite profiles created for ${ }^{1} \mathrm{H}$ NMR data: a 2D PCA, b 3D PCA and $\mathbf{c}$ OPLS-DA scores plots of the tumor (red) and normal (green) tissue samples. d The OPLS-DA loading S-plot showing the distribution patterns of metabolites to the differences between control and tumor samples
A

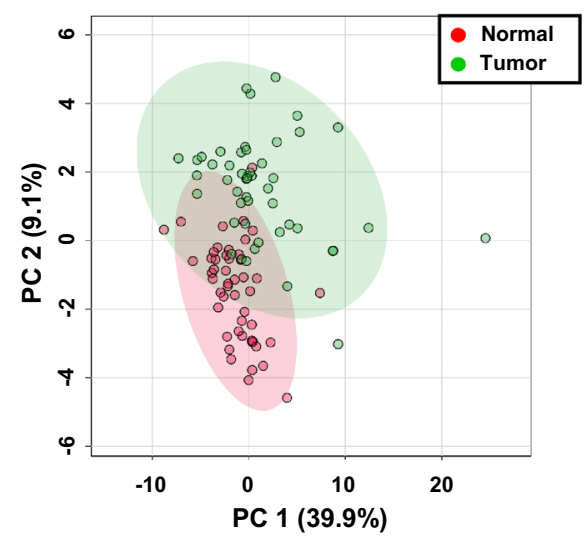

C

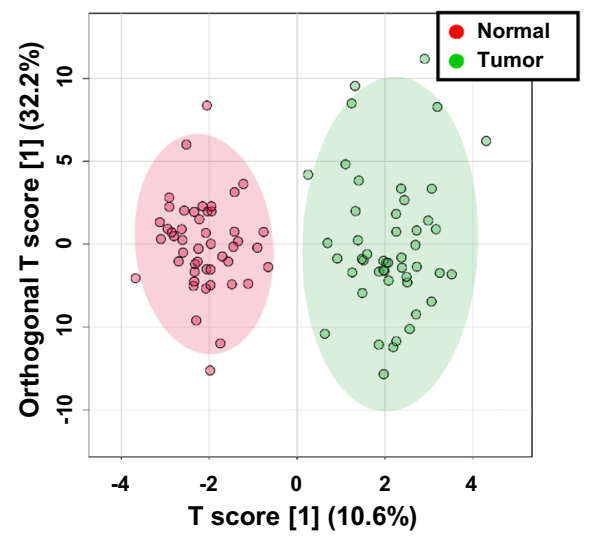

B

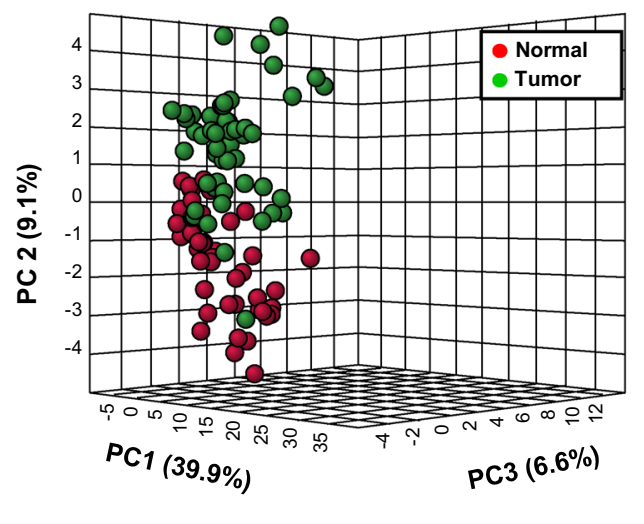

D

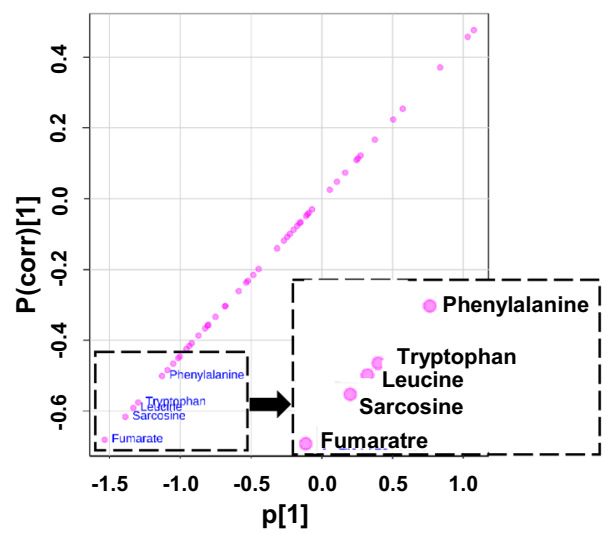

substantial differences in metabolic profiles of cancer and normal renal tissues (Fig.S1, Supplementary material).

An OPLS-DA statistical method referred to as S-plot was employed to identify metabolites present in significantly different levels in kidney tumors and adjacent normal tissues. S-plot of kidney tumor tissues vs. control was shown in Fig. 2d. Higher values of $\mid p($ corr)l indicate metabolites that are more important to the classification. Variables with $\mid p$ (corr)| value greater than 0.5 were considered significant. A paired t-test was performed for all variables. Five variables (listed in Table 1) were negatively correlated to group separation showing $\mathrm{p}$ (corr)[1] score of $<-0.5$. Analysis of the S-score of the OPLS-DA model, combined with statistical paired t-test analysis ( $\mathrm{p}$ values $<0.05$ ) indicate these five metabolites responsible for differences (Fig. 2c; Table 1). OPLS-DA analysis revealed statistically lower levels of fumarate, leucine, sarcosine and phenylalanine in the cancer tissues compared to adjacent control tissues. Concentration data for the set of 48 metabolites with mean concentrations statistical parameters are reported in Table S3 (Supplementary material).

Next, univariate ROC curves were generated to characterize the predictive value of selected metabolites independently. The quality of the ranking represents the area under the curve (AUC). The results of univariate ROC curve analyses indicated that in the tissue samples, all five previously selected metabolites (fumarate, leucine, sarcosine, tryptophan and phenylalanine) exhibit high AUC above 0.78. Among these metabolites, the best ROC analyses with highest significance were achieved for fumarate $(\mathrm{AUC}=0.918$, specificity $=0.8$ and a sensibility $=0.9)$, followed by leucine $(\mathrm{AUC}=0.898$, specificity $=0.9$ and a sensibility $=0.9)$, sarcosine $(\mathrm{AUC}=0.887$, specificity $=0.9$ and a sensibility $=0.9)$ and tryptophan $(\mathrm{AUC}=0.857$, specificity $=0.8$ and a sensibility $=0.9$ ) The range of concentrations for two first individual metabolites in the tissue samples of cancer patients compared to normal controls is shown in Fig. 3a and b.

A multivariable ROC analysis was performed to further assess the predictive value of these metabolites as a group to discriminate between tumor and normal tissues of each patient. The classification model was built using the MetaboAnalyst software and it's associated the random forest algorithm. Figure $3 \mathrm{c}$ demonstrated that using a combination of metabolite level changes was a better discriminator (AUC > 0.94) than comparing each metabolite level 
Table 1 Summary of the subsets of potential biomarkers of kidney cancer (p-value $<0.05 ; \mid p($ corr $) \mid>0.5)$

\begin{tabular}{|c|c|c|c|c|c|c|c|c|c|}
\hline $\begin{array}{l}\text { Comparison } \\
\text { mode }\end{array}$ & Data set & $\begin{array}{l}\text { Metabolite/ele- } \\
\text { ment }\end{array}$ & $m / z^{\mathrm{a}}$ & Adduct type & $\begin{array}{l}\text { Mass error } \\
{[\mathrm{ppm}]}\end{array}$ & AUC & $\mathrm{p}$ (corr) & P-value ${ }^{b}$ & Fold change $^{c}$ \\
\hline \multirow{5}{*}{$\begin{array}{l}\text { Normal vs. } \\
\text { Tumor }\end{array}$} & \multirow[t]{5}{*}{${ }^{1} \mathrm{H}$ NMR } & Fumarate $^{\mathrm{d}}$ & & & & 0.91 & -0.681 & $4.1 \mathrm{E}-11$ & 3.1 \\
\hline & & Leucine $^{\mathrm{d}}$ & & & & 0.90 & -0.592 & $2.6 \mathrm{E}-11$ & 2.2 \\
\hline & & Sarcosine $^{d}$ & & & & 0.89 & -0.617 & $7.7 \mathrm{E}-09$ & 3.8 \\
\hline & & Tryptophan $^{\mathrm{d}}$ & & & & 0.86 & -0.576 & $3.9 \mathrm{E}-08$ & 2.5 \\
\hline & & Phenylalanine $^{\mathrm{d}}$ & & & & 0.78 & -0.501 & $6.1 \mathrm{E}-06$ & 1.8 \\
\hline \multirow{2}{*}{$\begin{array}{l}\text { Benign vs. } \\
\text { Malignant }\end{array}$} & \multirow[t]{2}{*}{${ }^{1} \mathrm{H}$ NMR } & Glucose $^{\mathrm{d}}$ & & & & 0.89 & 0.609 & $2.96 \mathrm{E}-05$ & 0.2 \\
\hline & & Creatine $^{\mathrm{d}}$ & & & & 0.77 & -0.575 & $7.36 \mathrm{E}-03$ & 3.9 \\
\hline \multirow{3}{*}{$\begin{array}{l}\text { Normal vs. } \\
\text { Tumor }\end{array}$} & \multirow[t]{3}{*}{ ICP-OES } & $\mathrm{Zn}^{\mathrm{d}}$ & & & & 0.94 & -0.866 & $7.99 \mathrm{E}-06$ & 2.4 \\
\hline & & $S^{\mathrm{d}}$ & & & & 0.84 & -0.750 & $2.59 \mathrm{E}-05$ & 1.2 \\
\hline & & $\mathrm{Na}^{\mathrm{d}}$ & & & & 0.73 & -0.510 & $2.47 \mathrm{E}-03$ & 1.2 \\
\hline \multirow[t]{11}{*}{$\begin{array}{l}\text { Normal vs. } \\
\text { Tumor }\end{array}$} & \multirow[t]{11}{*}{${ }^{109} \mathrm{Ag}$ LDI MS } & $\begin{array}{l}\text { Hydroxyeicosa- } \\
\text { trienoic acid }\end{array}$ & 361.208 & {$\left[\mathrm{C}_{20} \mathrm{H}_{34} \mathrm{O}_{3}+\mathrm{K}\right]^{+}$} & -15.5 & 0.79 & -0.569 & $7.84 \mathrm{E}-06$ & 8.7 \\
\hline & & $U^{f}$ & 121.122 & - & - & 0.78 & -0.586 & $1.34 \mathrm{E}-05$ & 42.3 \\
\hline & & Octanediol $^{\mathrm{e}}$ & 147.137 & {$\left[\mathrm{C}_{8} \mathrm{H}_{18} \mathrm{O}_{2}+\mathrm{H}\right]^{+}$} & -6.5 & 0.77 & -0.524 & $2.36 \mathrm{E}-05$ & 6.9 \\
\hline & & $\begin{array}{l}\text { Diethoxypen- } \\
\text { tane } \mathrm{e}^{\mathrm{e}}\end{array}$ & 161.153 & {$\left[\mathrm{C}_{9} \mathrm{H}_{20} \mathrm{O}_{2}+\mathrm{H}\right]^{+}$} & -3.8 & 0.77 & -0.501 & $7.84 \mathrm{E}-06$ & 8.3 \\
\hline & & $\mathrm{UN}^{\mathrm{f}}$ & 243.824 & - & - & 0.77 & 0.518 & $1.03 \mathrm{E}-05$ & 0.8 \\
\hline & & $\mathrm{UN}^{\mathrm{f}}$ & 331.568 & - & - & 0.75 & -0.522 & $5.46 \mathrm{E}-05$ & 2.8 \\
\hline & & $\mathrm{UN}^{\mathrm{f}}$ & 120.940 & - & - & 0.76 & -0.516 & $9.39 \mathrm{E}-06$ & 1.7 \\
\hline & & Oxoalanine $^{\mathrm{d}}$ & 141.991 & {$\left[\mathrm{C}_{3} \mathrm{H}_{5} \mathrm{NO}_{3}+\mathrm{K}\right]^{+}$} & 6.3 & 0.84 & -0.568 & $8.95 \mathrm{E}-07$ & 4.0 \\
\hline & & $U^{f}$ & 142.057 & - & - & 0.75 & 0.572 & $6.54 \mathrm{E}-06$ & 0.4 \\
\hline & & $\begin{array}{l}\text { 1-(Methyl- } \\
\text { thio)ethyl- } \\
\text {-2-propenyl } \\
\text { disulfide }^{\mathrm{e}}\end{array}$ & 181.015 & {$\left[\mathrm{C}_{6} \mathrm{H}_{12} \mathrm{~S}_{3}+\mathrm{H}\right]^{+}$} & -13.2 & 0.75 & 0.569 & $2.35 \mathrm{E}-06$ & 0.3 \\
\hline & & $\mathrm{UN}^{\mathrm{f}}$ & 491.781 & - & - & 0.75 & 0.560 & $3.31 \mathrm{E}-05$ & 0.3 \\
\hline
\end{tabular}

${ }^{a}$ Experimental monoisotopic mass; ${ }^{b}$-value determined from Student's t-test, ${ }^{c}$ fold change between normal and tumor tissue calculated from the tissue weight-normalized concentration (NMR and ICP-OES data) or abundance (LDI MS data) mean values for each group; ${ }^{\mathrm{d}}$ Identified com-

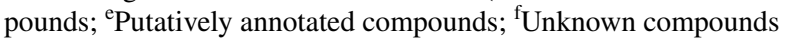

change separately. An excellent discriminating classification was obtained when the three metabolites sarcosine, fumarate and leucine (AUC of 0.962) were considered all together, with a confidence interval (CI) from 0.904 to 0.999 (Fig. 3d). ROC curve illustrating the performance of the NMR models in distinguishing between tumor and normal tissue using random forest algorithm on five chosen potential metabolite biomarkers is shown in Fig. S2 (Supplementary material). AUC of these five compounds was 0.969 demonstrating high specificity and sensitivity to distinguish the groups. The permutation test with predictive accuracy as performance measure and 1000 permutations showed a $p$ value $<0.001$. The average of predicted class probabilities of each sample and the average accuracy from predictive accuracy test of the ROC curve show a good classification of samples. Most of the samples were classified in their respective group (Fig. S2, Supplementary material). These results suggest that assessing the levels of these three specific metabolites in kidney tissue could significantly increase the diagnostic performance of this model and could be used as diagnostic biomarkers to distinguish with high specificity and sensitivity, cancerous from healthy tissue in patients diagnosed with kidney cancer.

\subsection{Distinguishing between type of kidney cancer with ${ }^{1} \mathrm{H}$ NMR}

${ }^{1} \mathrm{H}$ NMR metabolomics analysis of tissue samples was employed to evaluate whether distinct metabolic trends allow to distinguish between types (benign and malignant) of kidney cancers. A 3D PCA scores plot revealing a good discrimination between benign and malignant cases is shown in Fig. 4a.

Comparing benign versus malignant tissue with OPLSDA revealed a very good discrimination between these two groups (Fig. 4b). Quality factors for this model were: $Q^{2}$ : 0.395 and $R^{2} Y$ : 0.701 with $p$ values based on permutation tests lower than 0.05 . Detailed results are shown in supplementary material Fig.S3 (Supplementary materials). 

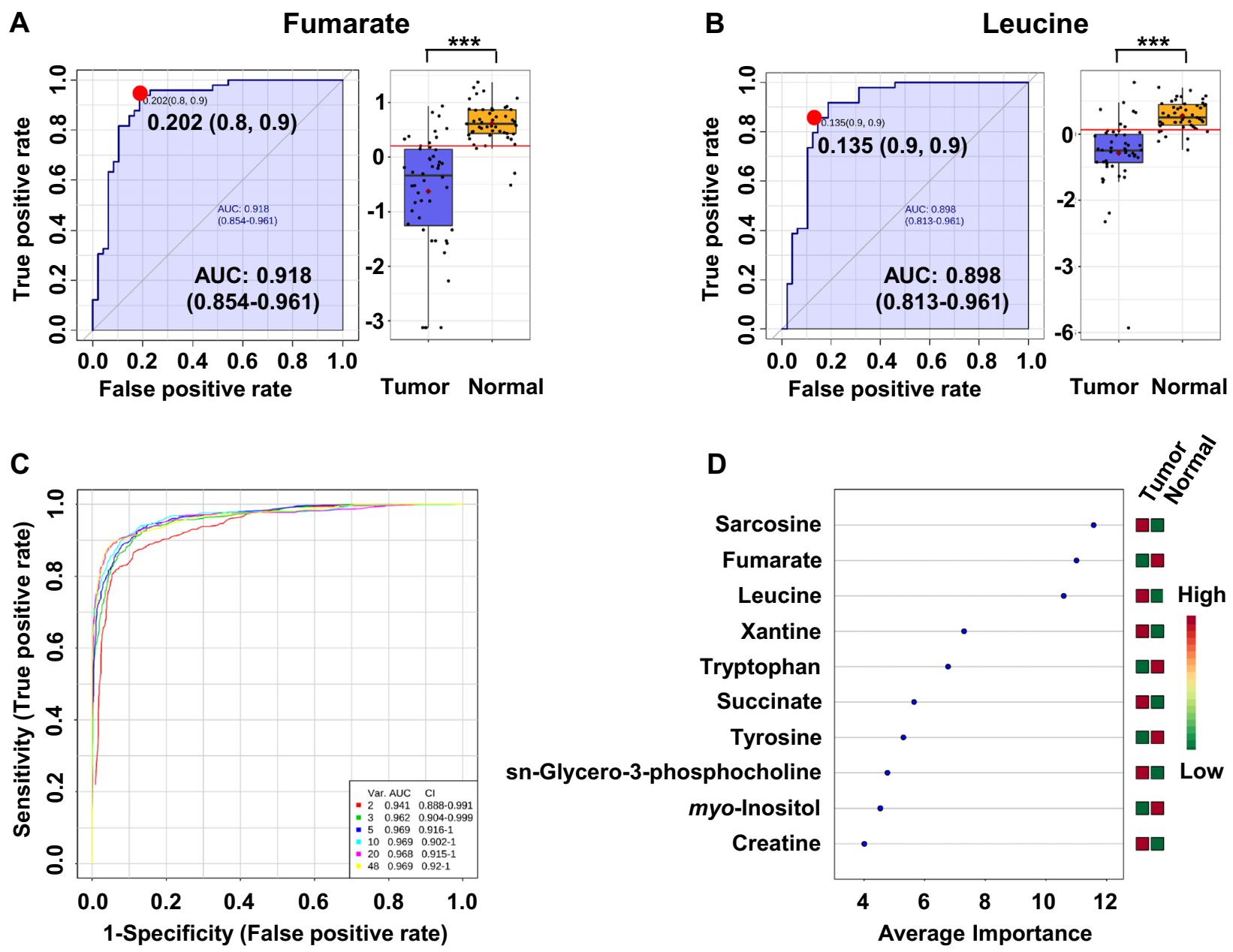

Fig. 3 ROC curves for the predictive model based on ${ }^{1} \mathrm{H}$ NMR data. a, b ROC curves of metabolites significantly associated with kidney cancer. c A combination of metabolites-model calculated with the

use of logistic regression analysis. d Metabolites with the highest ability to discriminate tumor kidney tissue against controls

Analysis of S-plots revealed that glucose and creatine are significant contributors to the separation between malignant vs benign samples (Fig. 4c, Table 2). In this study glucose was positively correlated with group separation with a p(corr)[1] score $>0.5$ and creatine negatively correlated with a $\mathrm{p}$ (corr) $[1]$ score $<-0.5$. Biomarker candidates were further subjected to t-test analysis to assess the significance of altered levels of these metabolites in benign versus malignant tissue samples. In total, 2 of the identified metabolites were found at statistically significant differential concentrations $(\mathrm{p}<0.05 ; \mathrm{q}<0.05$ and $\mid \mathrm{p}$ (corr) $\mid>0.5$ ), suggesting that examining the differential levels of glucose and creatine may be an effective way to identify malignant tissue and discriminate from healthy tissue within tissue samples of kidney cancer patients. Univariable ROC curve analyses were conducted to further assess the predictive value of these metabolite level changes. AUC values for glucose were found to be as high as 0.894 (Fig. 4d) and 0.769 for creatine (Fig. 4c).

Additionally, ROC curve for only these two selected metabolites were constructed with an AUC value of 0.889 (Fig. S4, Supplementary material). The permutation based on measure area under ROC curve test showed a p value $=0.003$. The average of predicted class probabilities of each sample across the 100 cross-validations indicates that most of the samples were classified in their respective group. The average accuracy based on 100 cross validations was 0.848 . These results support that glucose and creatine may be good discriminating indicators of kidney tumor types.

\subsection{Elemental profile of tissue in kidney cancer with ICP-OES}

Chemical elements concentrations obtained from ICP-OES experiments on 58 extracts of kidney tissue samples (28 cancer and 28 control tissues) were subjected to statistical data analysis. Elemental mean concentrations in tumorous 
A

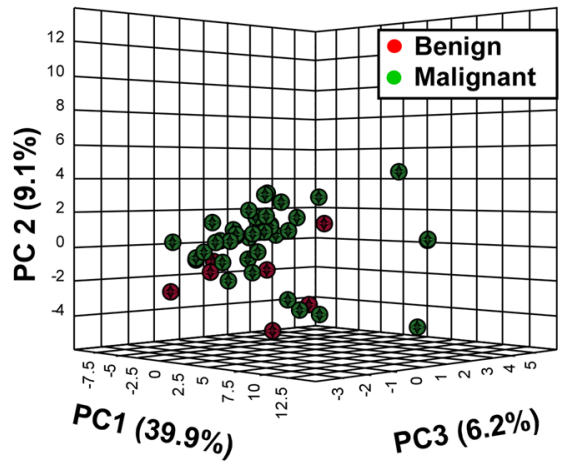

B

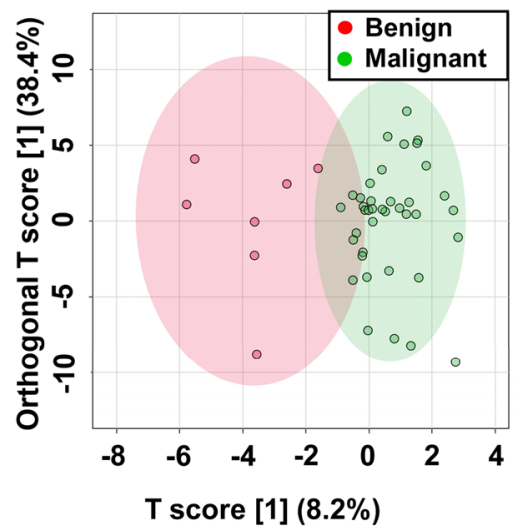

C

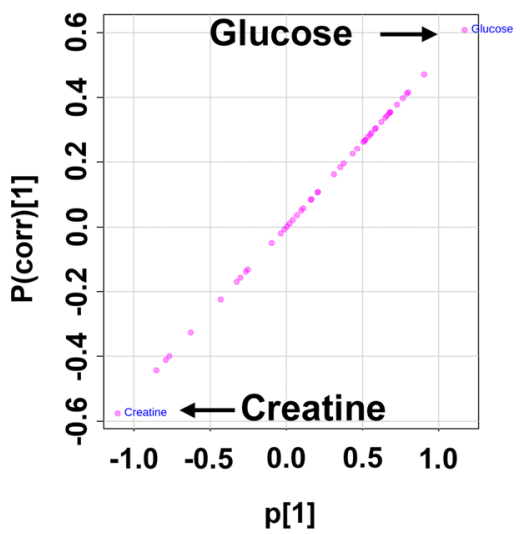

D

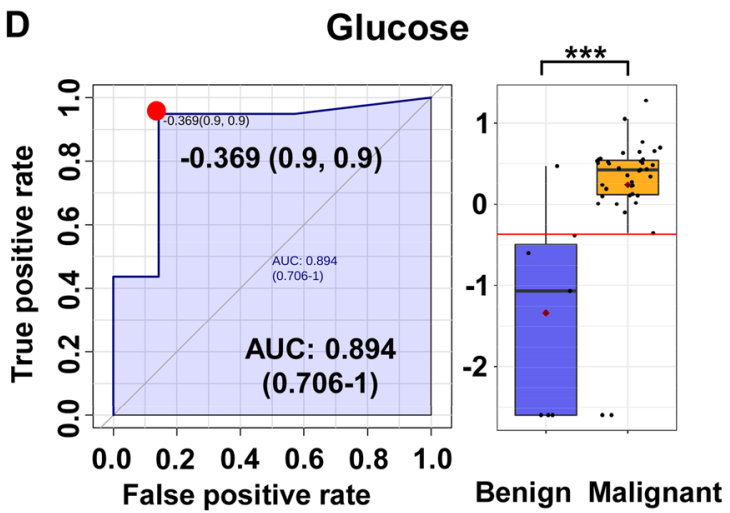

Fig. 4 Discrimination between benign and malignant kidney cancer based on the metabolic profiles of kidney tissue analyzed by ${ }^{1} \mathrm{H}$ NMR spectroscopy. a 3D PCA and b OPLS-DA scores plots generated from the NMR data of the benign (red) and malignant (green) tumors.

and non-tumorous tissues are summarized in Table S4 (Supplementary material) for each element. As shown in Fig. 5a, the 3D-PCA scores plot reveals a separation trend between the two groups. Results from OPLS-DA analysis, shown in Fig. 5b, provide a much clearer separation (compared to the PCA analysis) between tumor and normal tissues with high explanative and predictive parameters of this model: $\mathrm{R}^{2} \mathrm{X}$ and $\mathrm{Q}^{2}$ were 0.753 and 0.649 respectively (Fig.S5, Supplementary material).

Elements of interest were selected from the S-plot profile, constructed from the OPLS-DA model (Fig. 5c). The loading S-plot revealed that three variables were negatively correlated with group separation showing $-\mathrm{p}(\mathrm{corr})[1]<-0.5$. According to $p$-values and $p$ (corr) scores, three discriminate variables ( $\mathrm{Na}, \mathrm{S}$ and $\mathrm{Zn}$ ) were found to be potentially good discriminators of in kidney tissues (Table 1). ROC analysis revealed that zinc exhibited the highest significance with an AUC value of 0.948 , sensitivity of 0.9 , and specificity of 0.9 (Fig. 5d), and sulfur exhibited an AUC value of 0.835, sensitivity of 0.8 , and specificity of 0.9 (Fig. 5e). Furthermore, ROC curve plot illustrating the performance of the

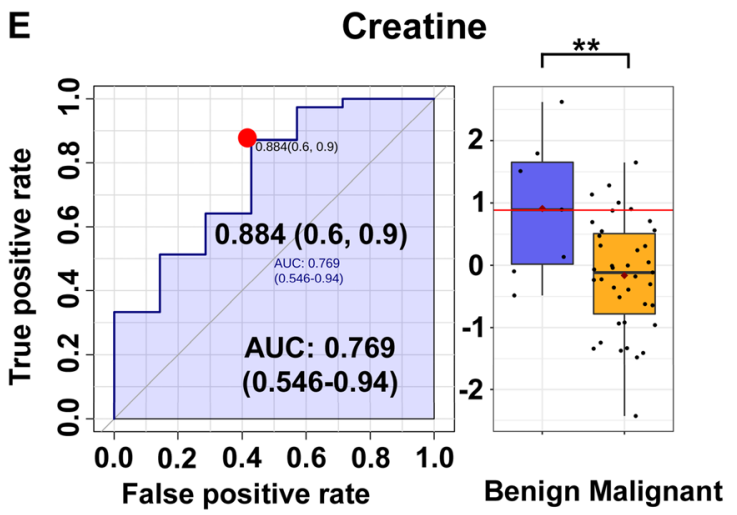

c The OPLS-DA loading S-plot showing the distribution patterns of metabolites to the differences between benign and malignant samples; d, e ROC curves of metabolites significantly associated with differentiation between types of kidney cancer

ICP OES model in distinguishing between tumor and normal tissue with only three selected elements ( $\mathrm{S}, \mathrm{Zn}, \mathrm{Na}$ ) was performed. AUC value was 0.953 which indicates very good discriminatory ability (Fig. S6, Supplementary material). The permutation based on area under ROC curve test showed a $\mathrm{p}$ value $<0.001$. The average of predicted class probabilities of each sample across the 100 cross-validations indicates that most of the samples were classified in their respective group. The average accuracy based on 100 cross validations was 0.899 . Most of the samples were classified in their respective group (Fig.S5, supplementary material). Levels of sulfur and sodium were identified as potentially good indicators of kidney cancer, while zinc concentration could be considered an excellent kidney cancer biomarker.

\subsection{Metabolic profiling of kidney cancer tissue with ${ }^{109}$ AgNPET LDI MS}

Laser mass spectrometry-based approach was also applied to investigate the tissue metabolic profiles of patients with kidney cancer. Statistical analysis was performed separately 
A

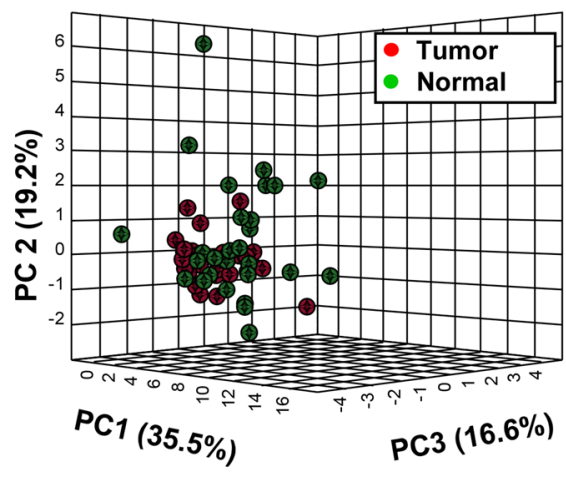

B

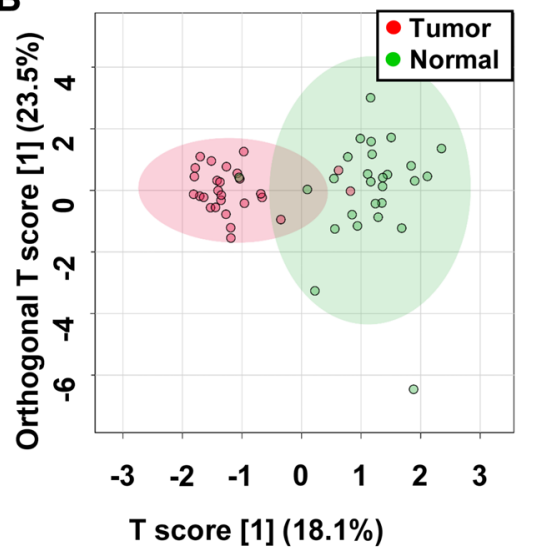

C

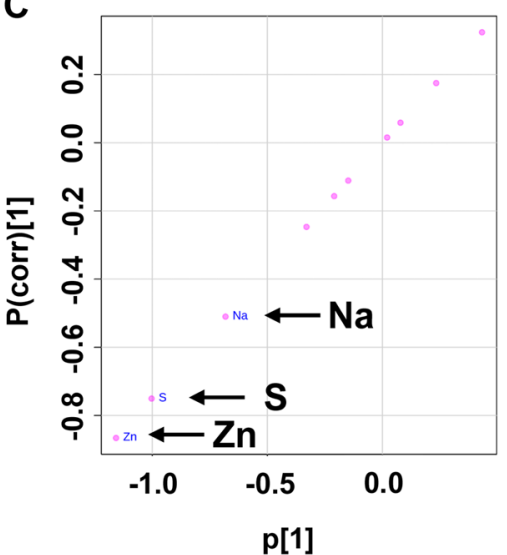

D

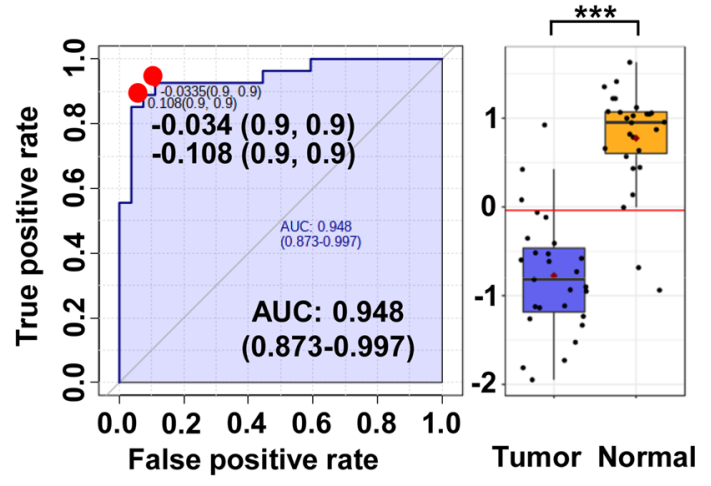

E

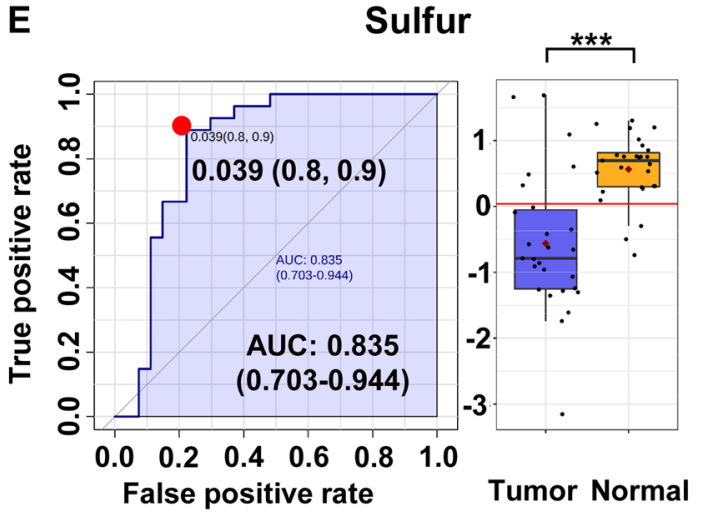

Fig. 5 Tissue metallomic profiles based on ICP-OES. a 3D PCA and $\mathbf{b}$ OPLS-DA scores plots generated from the ICP-OES data of the tumor tissue (red) and adjacent control tissue (green) samples. c The OPLS-DA loading S-plot showing the distribution patterns of

for polar and non-polar metabolite extracts of kidney tissue. 575 and 461 common features were detected in the non-polar and polar extracts of tissues samples, respectively of 50 patients with kidney cancer by ${ }^{109}$ AgNPET LDI mass spectral analyses. Spectral intensity data from LDI MS spectra was subjected to multivariate data analysis to assess whether these could discriminate between tissue types. 2D-PCA and OPLS-DA score plots were generated for the entire data sets, and 2D-PCA scores plots of mass spectral features revealed moderate discrimination between tumor and normal tissue (Fig.S7, supplementary material). Results from OPLS-DA analysis, shown in Fig.S7B, D (supplementary material), presented a clear separation between two groups suggesting that the ${ }^{109} \mathrm{AgNPET}$ LDI MS-based tissue metabolomics model can also be used to effectively identify discriminating metabolic differences that separate kidney tumor and normal tissues. All relevant spectral features and observations resulting from the ${ }^{109} \mathrm{AgNPET}$ LDI MS-based metabolomics analyses are reported in Table 1. elements to the differences between tumor and control tissue samples; d, $\mathbf{e}$ ROC curves of chosen elements significantly associated with kidney cancer

Potential features for group separation of non-polar and polar extracts of tissue samples were subsequently identified by S-plot loading analysis of corresponding OPLS-DA models, and based on significance criterion of $\mid p($ corr $) \mid>0.5$ and $|p|>0.05$ (Fig.S8 A, D, Supplementary material). The S-plot loading for the non-polar extract analysis revealed one variable as positively correlated with group separation, exhibiting $\mathrm{a}+\mathrm{p}$ (corr)[1] value $>0.5$ and five variables as negatively correlated with group separation, and exhibiting $\mathrm{a}-\mathrm{p}$ (corr) [1] value $<-0.5$. Resulting $R^{2} Y$ and $Q^{2}$ parameters assessing the validity of the OPLS-DA model consisted of 0.905 (p-value $<5 \mathrm{E}-04(0 / 2000)$ ) and 0.337 (p-value $<5 \mathrm{E}-04)$, respectively (Fig. S8 B, Supplementary material). Mean abundances of spectral features identified in the non-polar extracts of tissue samples are reported in Table S5 (Supplementary material). The S-plot loading for the polar extracts revealed three variables as positively correlated with group separation and $+p$ (corr) $[1]>0.5$, and two negatively correlated with group separation and $-\mathrm{p}$ (corr) $[1]<-0.5$. Resulting $R^{2} Y$ and $Q^{2}$ values were respectively 0.905 
(p-value $<5 \mathrm{E}-04(0 / 2000))$ and 0.337 (p-value $<5 \mathrm{E}-04)$, providing a robust indication of goodness of fit and predictability of the two-class OPLSDA model (Fig.S8 E, Supplementary material). Mean abundances of $m / z$ variables identified to be significantly different in the non-polar extracts of tumor vs normal tissue samples are reported in Table S6 (Supplementary material).

Selected $\mathrm{m} / \mathrm{z}$ values were subjected to multivariate ROC curve analyses based on random forest algorithms. All eleven previously selected $\mathrm{m} / \mathrm{z}$ mass spectral features were identified to exhibit high AUC values with an area under the curve $>0.75$ (Fig.S9, Supplementary information). This analysis was followed by a multivariate ROC analysis to assess whether groups of spectral features were better predictors compared to when features were analyzed separately. As shown in Figures S10 (Supplementary material), the combination of mass features in non-polar extracts was found to be a better discriminator between healthy and malignant tissue than the individual analysis of each mass feature. A good classification was obtained with ten features (AUC of 0.82), with confidence intervals (CI) ranging from 0.729 to 0.906 . A good classification was also obtained for spectral features from polar extracts of kidney tissue, with ten of these features exhibiting AUC values of 0.818 and CI ranging from 0.705 to 0.911 . For both MS models features with the highest ability of contributions to classification accuracy are shown in Fig.S10 B and D (Supplementary material). These results suggest that selected mass features can significantly increase the diagnostic performance of MS model and could be used as diagnostic biomarkers that separate cancer tissue samples from normal with high specificity and sensitivity.

ROC curves illustrating the performance of the ${ }^{109} \mathrm{AgNPET}$ LDI MS model in distinguishing between tumor and normal tissue using random forest algorithm on six selected features from non-polar and five features from polar extracts of studied tissues were presented in figures S11 and S12 (Supplementary material). Putative identifications were guided by searches on various metabolite databases i.a. HMDB (Wishart et al., 2007), MetaCyc (Caspi et al., 2018), LipidMaps (Sud et al., 2007), and Metlin (Smith et al., 2005). Five tissue mass features were putatively identified as known metabolites. All important mass spectral features and metabolite ID resulting from the ${ }^{109} \mathrm{Ag}$ LDI MS analyses are reported in Table 1.

\subsection{Distinguishing between age and gender}

To evaluate the significant changes between tissue extracts from patients of different sex and age the analysis of variance (ANOVA) was performed. The analysis of gender and age differentiation was performed for data obtained from all three analytical platforms. The analysis was performed using samples in four groups; for gender discrimination samples from female, female control, male and male control were used. For age discrimination samples from patients of age under 60 and over 60 were used. Data was presented in supplementary materials (Figures S13-S18). However, in all cases, no statistically significant differences were observed between patients of different sex and age. It has been observed that data from tumor tissue extracts within each group (male vs female and age $>60 v s$ age $<60$ ) differed only compared to the adjacent extracts of control normal tissues. This is mainly due to the insufficient number of patients under 60 and of the female sex because the population studied was relatively old and mainly male.

\subsection{Pathway analysis of potential biomarkers}

The most differentiating metabolites between kidney cancer and normal tissues among three analytical platforms were subjected to pathway analysis by MetaboAnalyst 5.0. However, only the compounds selected in the NMR analysis turned out to be important in the metabolic pathways in the human body, therefore the quantitative data from this platform was used to identify the most relevant pathways involved in the kidney cancer. The concentrations of fumarate, sarcosine, leucine, tryptophan and phenylalanine were subjected to pathway analysis and quantitative enrichment analysis using a MetaboAnalyst 5.0. 12 metabolic pathways, including tyrosine metabolism, arginine and proline metabolism, purine metabolism, citric acid cycle, urea cycle, aspartate metabolism, mitochondrial electron transport chain, Warburg effect, phenylalanine and tyrosine metabolism, valine, leucine and isoleucine degradation, glycine and serine metabolism, methionine metabolism and tryptophan metabolism were significantly related to kidney cancer. The result of pathway analysis is shown in Fig. 6a and Table S7 in Supplementary materials. Furthermore, in order to expand metabolomic analysis of pathway related to kidney cancer, the quantitative enrichment analysis module in MetaboAnalyst, with extensive list of pathways from SMPDB database was performed. Concentrations of 5 metabolites, identified from the global metabolomic profiling, were entered as input data. It was found that 6 additional pathways including aspartate metabolism, methionine metabolism, mitochondrial electron transport chain, purine metabolism, urea cycle and Warburg effect significantly related to kidney cancer (Fig. 6b; Table S8, Supplementary materials). The power of metabolic pathways analysis was confirmed by p-value Holm p-values and FDR of less than 0.001 for every pathway (Tables S7 and S8, Supplementary material). 


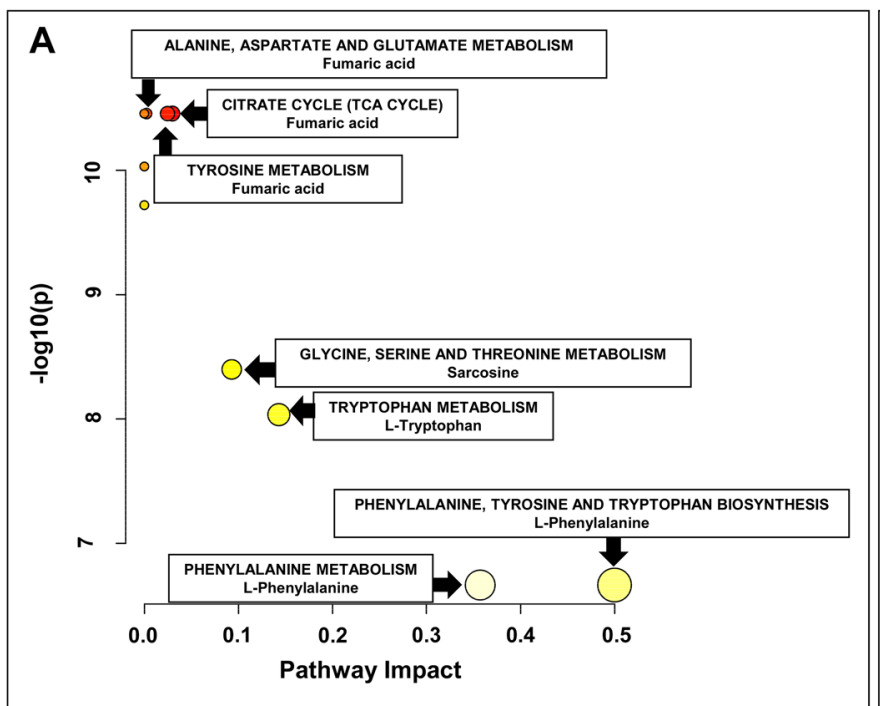

Fig. 6 Results of pathway topology analysis of selected five differential metabolites statistically significant in RCC (found in NMR spectra). a Pathway analysis based on KEGG; bubble area donating to the

\section{Discussion}

In this study, ${ }^{1} \mathrm{H}$ NMR, ICP-OES and ${ }^{109} \mathrm{Ag}$ LDI MS were employed to assess similarities and differences in metabolite and selected chemical elements abundances between renal tumors and normal parenchymal tissue. ${ }^{1} \mathrm{H}$ NMR metabolomics analysis revealed significant differences in the concentration of essential amino acid, including leucine, tryptophan and phenylalanine. Essential amino acids cannot be synthetized de novo by the human cells thus must be provided by diet and intestinal microbiota. Rapid and uncontrolled proliferation of cancer requires increased levels of amino acids to be used as basic building blocks for the synthesis of new proteins. In this study, depletion of essential amino acids is reflected in decreased concentration of leucine, tryptophan and phenylalanine in cancer tissue. Our results are consistent with the findings of Jing et. al. who also showed decreased level of numerous amino acids in RCC tissue specimens (Jing et al., n.d.).

Decreased levels of tryptophan may also be associated with its excessive consumption. Tryptophan metabolism plays a major role in cancer resistance to immunotherapeutic treatment, as it is metabolized to kynurenine via the kynurenine pathway. Kynurenine activates the transcription factor aryl hydrocarbon receptor (AhR) which induces immunosuppression by disrupting the ability of dendritic cells and T cells to eliminate cancer cells. This may also partially explain the high rate of IFN $\alpha$ therapy failure in RCC (Trott et al., 2016). Another amino acid derivative whose concentration was lower in RCC specimens is sarcosine, which can be converted to glycine by action of sarcosine

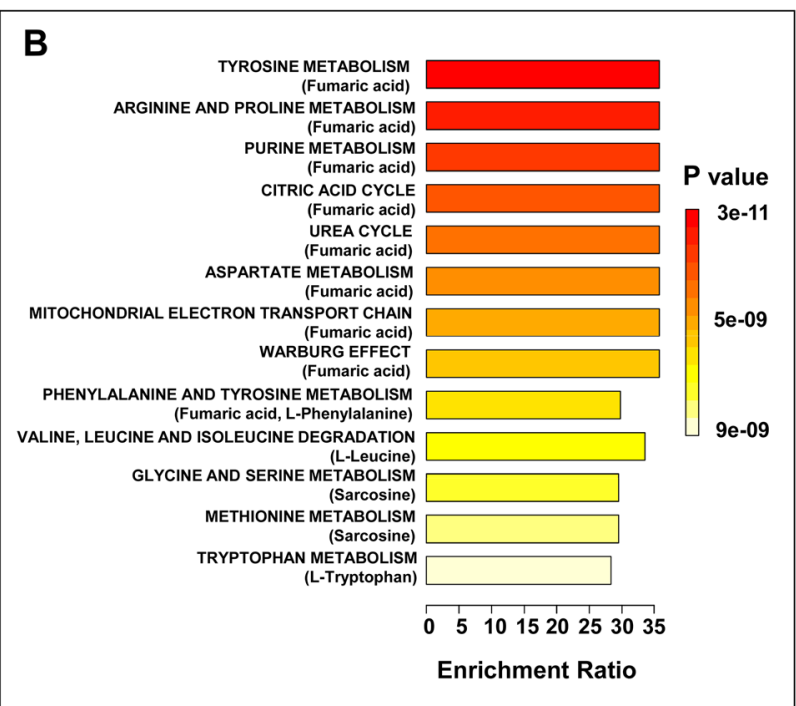

impact of each pathway with color representing the significance from highest in red to lowest in white; b Quantitative enrichment analysis based on SMPDB

dehydrogenase. It has been reported that elevated levels of sarcosine can be detected in the urine of the patients with prostate cancer (Sreekumar et al., 2009). However further studies failed to prove its value in prostate cancer diagnosis (Jentzmik et al., 2010).

ICP-OES analysis was conducted to assess potential differences in chemical elements abundance between cancer and control tissues. ICP-OES measurements revealed decreased concentrations of $\mathrm{Zn}, \mathrm{S}$ and $\mathrm{Na}$ in the tissue of renal tumors. Numerous elements have been implicated in the process of tumorigenesis, as heavy metals like arsenic, cadmium, chromium, and nickel are considered carcinogenic in humans (Kim et al., 2015). On the other hand, metals like zinc ( $\mathrm{Zn})$ and sodium (Na) are considered essential for normal human body functions. Zinc is the second most abundant metal in the human body, and is essential for over 300 enzyme functions including carbonic anhydrase, superoxide dismutase and alkaline phosphatase. Moreover, zinc is important in DNA and RNA metabolism, signal transduction, gene expression and protein folding via action of zinc finger motifs. This study demonstrated a decreased concentration of zinc in kidney tumors when compared to normal renal parenchyma. Moreover, zinc showed highest predictive value of all detected elements/metabolites with AUC of 0.948. Two studies evaluating the zinc level in the kidney tissue reported decreased levels of zinc in cancer samples. However, this relation was found to be statistically non-significant due to the small group size of the studies (Dobrowolski et al., 2002). Studies by Abdel-Gawad, Reddy and Calvo reported a statistically significant increase in zinc concentration in RCC specimen (Abdel-Gawad et al., 2020; 
Calvo et al., 2009; Reddy et al., 2003). On the other hand, studies by Dobrowolski et al. which evaluated zinc levels in kidney tissue reported a decreased level of zinc in cancer samples (Dobrowolski et al., 2002), providing cofounding results as to zinc level trends in cancer tissue.

Association between high concentration of heavy metals in cancer tissue and cancerogenesis have been extensively studied. Most studies agree that exposure to certain heavy metals, particularly arsenic, nickel, cadmium, chromium may increase the risk of cancer. Unfortunately, the causative mechanism is still unknown. Some studies hypothesize that heavy metals may induce cellular hypoxia through activation of hypoxia inducible factor 1 (Galanis et al., 2009; Osipyants et al., 2018). This may be important concerning pharmacological treatment of renal cancer which is based on the inhibition of HIF-1 induced overexpression of the products of tyrosine kinase incl. VEGF and PDGF. First line treatment of metastatic RCC is based on targeted therapy against tyrosine kinase and include tyrosine kinase inhibitors (sunitynib, pazopanib, axitinib, cabozantinib, lenvatinib and tivozanib) and monoclonal antibodies targeting VEGF (bevacizumab) (Ljungberg et al., 2007) Therefore, it may be hypothesized that chelation of heavy metals which may act as HIF-1 activators could improve efficacy of the treatment. Another important question is why elevated concentration of heavy metals in cancer tissue is observed. Romaniuk et al. suggested that this may be due to an impaired excretion of metabolites and heavy metals by cancer cells (Romaniuk et al., 2017). However, in this study, we observed decreased concentration of metals like sodium and zinc in cancer tissue. Sodium is the primary cation in the extracellular fluid and its main role is in regulation of water homeostasis between cells and extracellular space. This is made possible through a variety of sodium channels that operate between extra and intracellular space. This observed decrease in concentration of sodium concentration may be caused by dysfunction of those channels. Decreased concentration of zinc in cancer tissue was also observed by Dobrowolski et al. (2002) (Kwiatek et al., 1996) Studies have reported that zinc exerts a cytotoxic effect on cancer cells (Costello \& Franklin, 2005). Therefore, the decreased concentration of zinc in cancer tissue may be explained by an attempt to avoid cytotoxic effects on the malignant cells. Diagnosis of renal cell carcinoma subtype is based on histopathological evaluation of resected/biopsied tissue. Heavy metals are ubiquitous in the human body and therefore are not specific for cancer tissue and therefore they cannot be utilized alone as cancer biomarkers. However, in combination with other metabolites they may provide unique information concerning prognosis and staging.

Sodium is the primary cation in the extracellular fluid (EF), which includes interstitial fluid-i.e. fluid surrounding cells and intravascular fluid (blood plasma). Blood plasma sodium level varies between 135 and $145 \mathrm{mmol} / \mathrm{L}$, whereas intracellular levels are maintained at $12 \mathrm{mmol} / \mathrm{L}$. Sodium's major role is the regulation of water homeostasis in the human body, and is also essential for mediating electrical signaling of neuronal cells. However, in solid tumors, like kidney cancer, impaired growth of new blood vessels leading to hypoxia and themetabolic reprogramming of cancer cells favoring aerobic glycolysis (Warburg effect) leads to the development of an intracellular acidic environment. To maintain intracellular $\mathrm{pH}$, excess $\mathrm{H}^{+}$must be secreted outside the cell or neutralized by importing bicarbonate $\left(\mathrm{HCO}_{3}{ }^{-}\right)$. These processes are mediated by $\mathrm{Na}^{+} / \mathrm{H}^{+}$antiport. systems and $\mathrm{Na}^{+}, \mathrm{HCO}_{3}{ }^{-}$co-transporters which are coupled to intracellular $\mathrm{Na}^{+}$transport (Stock \& Pedersen, 2017). In this study we observed decreased concentration of sodium within kidney tumor tissue, reflecting changes in sodium levels in both extracellular and intracellular space. However, the intracellular volume fraction accounts for $80 \%$ of total tissue volume (Madelin \& Regatte, 2013). Therefore, this concentration decrease reflects mainly a decrease of sodium in intracellular space, which could be due to impairment of sodium channel function in cancer cells.

${ }^{109} \mathrm{Ag}$ LDI MS analysis revealed significance differences in abundance of 12 metabolites between kidney tumors and control. Six of them were putatively identified as: hydroxyeicosatetraenoic acid, octanediol, diethoxypentane, oxoalanine, 1-(methylthio)ethyl-2-propenyl disulfide, hydroxyeicosatetraenoic acid, octanediol, diethoxypentane and oxoalanine were found to be in higher concentration in cancer tissue whereas 1-(methylthio)ethyl-2-propenyl disulfide was higher in normal renal tissue.

Hydroxyeicosatetraenoic acid is an eicosanoid that is produced by enzymatic oxidation of arachidonic acid. It has been reported that 20-hydroxyeicosatetraenoic acid (20-HETE) is associated with cancerogenesis. Moreover, selective inhibitors of the 20-HETE-producing enzymes (CYP4A and CYP4F) can inhibit growth of numerous cell lines including renal cell carcinoma (Alexanian \& Sorokin, 2013). These findings are consistent with our observations that renal tumors contain higher levels of hydroxyeicosatetraenoic acid compared to normal renal tissue.

In this study glucose and creatine concentration were shown to discriminate between benign and malignant kidney tumors, with higher glucose concentrations found in cancerous tissue. Numerous studies have reported the accumulation of glucose in RCC tumors as a result of the metabolic reprogramming of RCC cells. It is believed that this is due to enhanced uptake of glucose resulting from overexpression of GLUT-1 glucose transporters under hypoxic environment (Lucarelli et al., 2015; Nakaigawa et al., 2017; Popławski et al., 2017). This hypothesis is supported by Chan et al. who showed that inhibition of GLUT-1 transporters starves RCC cells by depleting glucose supply (Chan et al., 2011). These 
observations also implicate that benign kidney tumors are more metabolically similar to normal tissue than malignant tumors.

In contrast to glucose, creatine was found in lower concentration in cancerous tissue. Creatine, a non-proteinderived amino acid, is crucial for energy storage as phosphocreatine is used to regenerate ATP. Study conducted on mice by Biase et al. showed that creatine uptake is important for the anti-tumor activities of CD8 T-cells (Di Biase et al., 2019). Lower concentration of creatine in cancer tissue may impair immune functions that are essential to fight cancer. In this study, a metabolite that the most discriminated between tumor and control tissue was fumarate. It was found in lower concentration in tumor tissue. The analysis of the biochemical pathways indicates that fumarate participates in ten metabolic pathways including alanine, aspartate and glutamate metabolism, arginine and proline metabolism, aspartate metabolism, citrate cycle, mitochondrial electron transport chain, purine metabolism, pyruvate metabolism, tyrosine metabolism, urea cycle and is also important in Warburg effect. Another metabolite, sarcosine was significant in pathways such as glycine, serine and threonine metabolism and methionine metabolism. Leucine participates in significantly changed KEGG and SMPDB pathways of aminoacyl-tRNA biosynthesis, valine, leucine and isoleucine biosynthesis and degradation. Significant KEGG and SMPDB pathway including aminoacyl-tRNA biosynthesis, phenylalanine, tyrosine as well as tryptophan biosynthesis are related to phenylalanine.

\section{Conclusion}

This work has demonstrated that value of high-resolution ${ }^{1} \mathrm{H}$ NMR, ICP-OES and ${ }^{109} \mathrm{AgNPET}$ LDI MS, along with multivariate statistics to characterize kidney tissue metabolome and metallome differences between tumor and normal tissue of patients suffering from kidney cancer. With regard to biomarker discovery, five potentially robust metabolic biomarkers in 49 tumor tissue samples of kidney cancer patients and 49 adjacent normal tissues treated as controls were identified using ${ }^{1} \mathrm{H}$ NMR spectroscopy, while 11 mass spectral features were identified from nanoparticle-based LDI mass spectrometry analyses. The most important endogenous compounds and trace elements having bioactive properties and pharmacological applicability were discussed in details. Moreover, we also demonstrated the possibility of discriminating between different kidney cancer types using ${ }^{1} \mathrm{H}$ NMR metabolomics. This study also supports the value of integrated NMR and mass spectrometry to identify candidate biomarkers and characteristic changes in small molecule metabolite levels which could prove to be very valuable for use as diagnostics or to track disease progression, offering less invasive ways to screen patients with kidney cancer.

Supplementary Information The online version contains supplementary material available at https://doi.org/10.1007/s11306-021-01779-2.

Author contributions Conceptualization: TR, JN; Methodology: JN, TR; Formal analysis: JN, TR; Investigation: JN, TR; Resources: KO, TR, BT; VC; LN; KN. Data Curation: VC, BT, JN, LN, KN, AA; Writing-Original Draft: JN, KO; Writing-Review \& Editing: JN, TR, VC, BT; Visualization: JN; Supervision: TR, VC, BT; Project Administration: TR; Funding acquisition: TR, VC, BT.

Funding Research was supported mainly by National Science Centre (Poland), research project OPUS Number 2016/23/B/ST4/00062. ${ }^{1} \mathrm{H}$ NMR spectra were recorded at Montana State University-Bozeman on a cryoprobe-equipped $600 \mathrm{MHz}(14 \mathrm{~T})$ AVANCE III solution NMR spectrometer housed in MSU's NMR Center. Funding for MSU NMR Center's NMR instruments has been provided in part by the NIH SIG program (1S10RR13878 and 1S10RR026659), the National Science Foundation (NSF-MRI:DBI-1532078), the Murdock Charitable Trust Foundation (2015066:MNL), and support from the office of the Vice President for Research, Economic Development, and Graduate Education at MSU.

Data availability The data that support the findings of this study is available from the corresponding author upon reasonable request.

\section{Declarations}

Conflict of interest The authors declare no competing financial and/or non-financial interests.

Consent to participate The patients provided written consent for participate in research.

Consent for publication The patients provided written informed consent for the publication of any associated data.

Ethical approval The study protocol was approved by local Bioethics Committee at the University of Rzeszow (Poland) (permission no. 2018/04/10).

Research involving human and/or animal participants This article does not contain any studies with human and/or animal participants performed by either of the authors.

Open Access This article is licensed under a Creative Commons Attribution 4.0 International License, which permits use, sharing, adaptation, distribution and reproduction in any medium or format, as long as you give appropriate credit to the original author(s) and the source, provide a link to the Creative Commons licence, and indicate if changes were made. The images or other third party material in this article are included in the article's Creative Commons licence, unless indicated otherwise in a credit line to the material. If material is not included in the article's Creative Commons licence and your intended use is not permitted by statutory regulation or exceeds the permitted use, you will need to obtain permission directly from the copyright holder. To view a copy of this licence, visit http://creativecommons .org/licenses/by/4.0/. 


\section{References}

Abdel-Gawad, M., Elsobky, E., Abdel-Hameed, M., Abdel-Rahim, M., Harraz, A., Shokeir, A. A., \& Ali-El-Dein, B. (2020). Quantitative and qualitative evaluation of toxic metals and trace elements in the tissues of renal cell carcinoma compared with the adjacent non-cancerous and control kidney tissues. Environmental Science and Pollution Research, 27(24), 30460-30467. https://doi. org/10.1007/s11356-020-09402-y

Alexanian, A., \& Sorokin, A. (2013). Targeting 20-HETE producing enzymes in cancer-Rationale, pharmacology, and clinical potential. Dove Press. https://doi.org/10.2147/OTT.S31586

Arendowski, A., Nizioł, J., \& Ruman, T. (2018). Silver-109-based laser desorption/ionization mass spectrometry method for detection and quantification of amino acids. Journal of Mass Spectrometry. https ://doi.org/10.1002/jms.4068

Bray, F., Ferlay, J., Soerjomataram, I., Siegel, R. L., Torre, L. A., \& Jemal, A. (2018). Global cancer statistics 2018: GLOBOCAN estimates of incidence and mortality worldwide for 36 cancers in 185 countries. CA: A Cancer Journal for Clinicians, 68(6), 394-424. https://doi.org/10.3322/caac.21492

Calvo, F. B., Santos, D., Rodrigues, C. J., Krug, F. J., Marumo, J. T., Schor, N., \& Bellini, M. H. (2009). Variation in the distribution of trace elements in renal cell carcinoma. Biological Trace Element Research, 130(2), 107-113. https://doi.org/10.1007/s1201 1-009-8325-x

Caspi, R., Billington, R., Fulcher, C. A., Keseler, I. M., Kothari, A., Krummenacker, M., et al. (2018). The MetaCyc database of metabolic pathways and enzymes. Nucleic Acids Research, 46(D1), D633-D639. https://doi.org/10.1093/nar/gkx935

Catchpole, G., Platzer, A., Weikert, C., Kempkensteffen, C., Johannsen, M., Krause, H., et al. (2011). Metabolic profiling reveals key metabolic features of renal cell carcinoma. Journal of Cellular and Molecular Medicine, 15(1), 109-118. https:// doi.org/10.1111/j.1582-4934.2009.00939.x

Chan, D. A., Sutphin, P. D., Nguyen, P., Turcotte, S., Lai, E. W., Banh, A., et al. (2011). Targeting GLUT1 and the Warburg effect in renal cell carcinoma by chemical synthetic lethality. Science Translational Medicine, 3(94), 94ra70. https://doi. org/10.1126/scitranslmed.3002394

Chong, J., Soufan, O., Li, C., Caraus, I., Li, S., Bourque, G., et al. (2018). MetaboAnalyst 4.0: towards more transparent and integrative metabolomics analysis. Nucleic Acids Research, 46(W1), W486-W494. https://doi.org/10.1093/nar/gky310

Costello, L. C., \& Franklin, R. B. (2005). 'Why do tumour cells glycolyse?': From glycolysis through citrate to lipogenesis. Molecular and Cellular Biochemistry, 280(1-2), 1-8. https:// doi.org/10.1007/s11010-005-8841-8

Di Biase, S., Ma, X., Wang, X., Yu, J., Wang, Y. C., Smith, D. J., et al. (2019). Creatine uptake regulates CD8 T cell antitumor immunity. Journal of Experimental Medicine, 216(12), 28692882. https://doi.org/10.1084/jem.20182044

Dobrowolski, Z., Drewniak, T., Kwiatek, W., \& Jakubik, P. (2002). Trace elements distribution in renal cell carcinoma depending on stage of disease. European Urology, 42(5), 475-480. https ://doi.org/10.1016/S0302-2838(02)00400-1

Galanis, A., Karapetsas, A., \& Sandaltzopoulos, R. (2009). Metalinduced carcinogenesis, oxidative stress and hypoxia signalling. Mutation research-Genetic toxicology and environmental mutagenesis. Elsevier. https://doi.org/10.1016/j.mrgen tox.2008.10.008

Gao, H., Dong, B., Jia, J., Zhu, H., Diao, C., Yan, Z., et al. (2012). Application of ex vivo $1 \mathrm{H}$ NMR metabonomics to the characterization and possible detection of renal cell carcinoma metastases. Journal of Cancer Research and Clinical Oncology, 138(5), 753-761. https://doi.org/10.1007/s00432-011-1134-6

Gao, H., Dong, B., Liu, X., Xuan, H., Huang, Y., \& Lin, D. (2008). Metabonomic profiling of renal cell carcinoma: High-resolution proton nuclear magnetic resonance spectroscopy of human serum with multivariate data analysis. Analytica Chimica Acta, 624(2), 269-277. https://doi.org/10.1016/j.aca.2008.06.051

Gupta, A., Nath, K., Bansal, N., \& Kumar, M. (2020). Role of metabolomics-derived biomarkers to identify renal cell carcinoma: A comprehensive perspective of the past ten years and advancements. Expert Review of Molecular Diagnostics. https://doi. org/10.1080/14737159.2020.1704259

Hsieh, J. J., Purdue, M. P., Signoretti, S., Swanton, C., Albiges, L., Schmidinger, M., et al. (2017). Renal cell carcinoma (RCC) encompasses a heterogeneous group of cancers derived from renal tubular epithelial cells. Nature Reviews Disease Primers. https://doi.org/10.1038/nrdp.2017.9

Jentzmik, F., Stephan, C., Miller, K., Schrader, M., Erbersdobler, A., Kristiansen, G., et al. (2010). Sarcosine in urine after digital rectal examination fails as a marker in prostate cancer detection and identification of aggressive tumours. European Urology, 58(1), 12-18. https://doi.org/10.1016/j.eururo.2010.01.035

Jing, L., Guigonis, J.-M., Borchiellini, D., Durand, M., Pourcher, T., \& Ambrosetti, D. (n.d.). LC-MS based metabolomic profiling for renal cell carcinoma histologic subtypes. https://doi.org/10.1038/ s41598-019-52059-y

Kim, H. S., Kim, Y. J., \& Seo, Y. R. (2015). An overview of carcinogenic heavy metal: Molecular toxicity mechanism and prevention. Journal of Cancer Prevention, 20(4), 232-240. https://doi. org/10.15430/jcp.2015.20.4.232

Kwiatek, W. M., Drewniak, T., Lekka, M., \& Wajdowicz, A. (1996). Investigation of trace elements in cancer kidney tissues by SRIXE and PIXE. Nuclear Instruments and Methods in Physics Research, Section B: Beam Interactions with Materials and Atoms, 109-110, 284-288. https://doi.org/10.1016/0168-583X(95)00923-X

Lim, W., Graves, A., Hessamodini, H., \& Wong, G. (2013). Metastatic renal cell carcinoma: Update on epidemiology, genetics, and therapeutic modalities. ImmunoTargets and Therapy. https:// doi.org/10.2147/itt.s31426

Lin, L., Huang, Z., Gao, Y., Chen, Y., Hang, W., Xing, J., \& Yan, X. (2012). LC-MS-based serum metabolic profiling for genitourinary cancer classification and cancer type-specific biomarker discovery. Proteomics, 12(14), 2238-2246. https://doi.org/10.1002/ pmic. 201200016

Ljungberg, B., Hanbury, D. C., Kuczyk, M. A., Merseburger, A. S., Mulders, P. F. A., Patard, J. J., \& Sinescu, I. C. (2007). Renal cell carcinoma guideline. In S. Raj (Ed.), European urology. Elsevier. https://doi.org/10.1016/j.eururo.2007.03.035

Lucarelli, G., Galleggiante, V., Rutigliano, M., Sanguedolce, F., Cagiano, S., Bufo, P., et al. (2015). Metabolomic profile of glycolysis and the pentose phosphate pathway identifies the central role of glucose-6-phosphate dehydrogenase in clear cell-renal cell carcinoma. Oncotarget, 6(15), 13371-13386. https://doi.org/10.18632 /oncotarget.3823

Madelin, G., \& Regatte, R. R. (2013). Biomedical applications of sodium MRI in vivo. Journal of Magnetic Resonance Imaging, 38(3), 511-529. https://doi.org/10.1002/jmri.24168

Mishra, S., Dwivedi, S. P., \& Singh, R. B. (2014). A review on epigenetic effect of heavy metal carcinogens on human health. The Open Nutraceuticals Journal, 3(1), 188-193. https://doi. org/10.2174/18763960010030100188

Moka, D., Vorreuther, R., Schicha, H., Spraul, M., Humpfer, E., Lipinski, M., et al. (1998). Biochemical classification of kidney carcinoma biopsy samples using magic-angle-spinning $1 \mathrm{H}$ nuclear magnetic resonance spectroscopy. Journal of Pharmaceutical and 
Biomedical Analysis, 17(1), 125-132. https://doi.org/10.1016/ S0731-7085(97)00176-3

Mulware, S. J. (2013). Trace elements and carcinogenicity: A subject in review. 3 Biotech, 3(2), 85-96. https://doi.org/10.1007/s1320 5-012-0072-6

Nakaigawa, N., Kondo, K., Ueno, D., Namura, K., Makiyama, K., Kobayashi, K., et al. (2017). The acceleration of glucose accumulation in renal cell carcinoma assessed by FDG PET/CT demonstrated acquisition of resistance to tyrosine kinase inhibitor therapy. $B M C$ Cancer, 17(1), 39. https://doi.org/10.1186/s12885-016-3044-0

Nizioł, J., Misiorek, M., \& Ruman, T. (2019). Mass spectrometry imaging of low molecular weight metabolites in strawberry fruit (Fragaria x ananassa Duch.) cv. Primoris with 109Ag nanoparticle enhanced target. Phytochemistry, 159, 11-19. https://doi. org/10.1016/J.PHYTOCHEM.2018.11.014

Nizioł, J., Ossoliński, K., Tripet, B. P., Copié, V., Arendowski, A., $\&$ Ruman, T. (2020). Nuclear magnetic resonance and surfaceassisted laser desorption/ionization mass spectrometry-based serum metabolomics of kidney cancer. Analytical and Bioanalytical Chemistry. https://doi.org/10.1007/s00216-020-02807-1

Nizioł, J., Ossoliński, K., Tripet, B. P., Copié, V., Arendowski, A., $\&$ Ruman, T. (2021). Nuclear magnetic resonance and surfaceassisted laser desorption/ionization mass spectrometry-based metabolome profiling of urine samples from kidney cancer patients. Journal of Pharmaceutical and Biomedical Analysis, 193, 113752. https://doi.org/10.1016/j.jpba.2020.113752

Nizioł, J., Rode, W., Zieliński, Z., \& Ruman, T. (2013). Matrix-free laser desorption-ionization with silver nanoparticle-enhanced steel targets. International Journal of Mass Spectrometry, 335, 22-32. https://doi.org/10.1016/J.IJMS.2012.10.009

Nizioł, J., Sunner, J., Beech, I., Ossoliński, K., Ossolińska, A., Ossoliński, T., et al. (2020). Localization of metabolites of human kidney tissue with infrared laser-based selected reaction monitoring mass spectrometry imaging and silver-109 nanoparticle-based surface assisted laser desorption/ionization mass spectrometry imaging. Analytical Chemistry, 92(6), 4251-4258. https://doi. org/10.1021/acs.analchem.9b04580

Osipyants, A. I., Smirnova, N. A., Khristichenko, A. Y., Nikulin, S. V., Zakhariants, A. A., Tishkov, V. I., et al. (2018). Metal ions as activators of hypoxia inducible factor. Moscow University Chemistry Bulletin, 73(1), 13-18. https://doi.org/10.3103/S002713141 8020128

Popławski, P., Tohge, T., Bogusławska, J., Rybicka, B., Tański, Z., Treviño, V., et al. (2017). Integrated transcriptomic and metabolomic analysis shows that disturbances in metabolism of tumor cells contribute to poor survival of RCC patients. Biochimica et Biophysica Acta-Molecular Basis of Disease, 1863(3), 744-752. https://doi.org/10.1016/j.bbadis.2016.12.011

Reddy, S. B., Charles, M. J., Raju, G. J. N., Vijayan, V., Reddy, B. S., Kumar, M. R., \& Sundareswar, B. (2003). Trace elemental analysis of carcinoma kidney and stomach by PIXE method. Nuclear Instruments and Methods in Physics Research, Section B: Beam Interactions with Materials and Atoms, 207(3), 345-355. https://doi.org/10.1016/S0168-583X(03)00463-4

Romaniuk, A., Lyndin, M., Sikora, V., Lyndina, Y., Romaniuk, S., \& Sikora, K. (2017). Heavy metals effect on breast cancer progression. Journal of Occupational Medicine and Toxicology, 12(1), 32. https://doi.org/10.1186/s12995-017-0178-1

Shim, E.-H., Livi, C. B., Rakheja, D., Tan, J., Benson, D., Parekh, V., et al. (2014). L-2-hydroxyglutarate: An epigenetic modifier and putative oncometabolite in renal cancer. Cancer Discovery, 4(11), 1290-1298. https://doi.org/10.1158/2159-8290.CD-13-0696

Smith, C. A., O’Maille, G., Want, E. J., Qin, C., Trauger, S. A., Brandon, T. R., et al. (2005). METLIN: A metabolite mass spectral database. Therapeutic Drug Monitoring, 27, 747-751. https://doi. org/10.1097/01.ftd.0000179845.53213.39

Sreekumar, A., Poisson, L. M., Rajendiran, T. M., Khan, A. P., Cao, Q., Yu, J., et al. (2009). Metabolomic profiles delineate potential role for sarcosine in prostate cancer progression. Nature, 457(7231), 910-914. https://doi.org/10.1038/nature07762

Stock, C., \& Pedersen, S. F. (2017). Roles of $\mathrm{pH}$ and the $\mathrm{Na}+\mathrm{H}+$ exchanger NHE1 in cancer: From cell biology and animal models to an emerging translational perspective. Seminars in cancer biology. Academic Press.

Sud, M., Fahy, E., Cotter, D., Brown, A., Dennis, E. A., Glass, C. K., et al. (2007). LMSD: LIPID MAPS structure database. Nucleic Acids Research. https://doi.org/10.1093/nar/gk1838

Tate, A. R., Foxall, P. J. D., Holmes, E., Moka, D., Spraul, M., Nicholson, J. K., \& Lindon, J. C. (2000). Distinction between normal and renal cell carcinoma kidney cortical biopsy samples using pattern recognition of $1 \mathrm{H}$ magic angle spinning (MAS) NMR spectra. NMR in Biomedicine, 13(2), 64-71. https://doi. org/10.1002/(SICI)1099-1492(200004)13:2\%3c64::AID-NBM61 2\%3e3.0.CO;2-X

Trott, J. F., Kim, J., Aboud, O. A., Wettersten, H., Stewart, B., Berryhill, G., et al. (2016). Inhibiting tryptophan metabolism enhances interferon therapy in kidney cancer. Oncotarget, 7(41), 66540 66557. https://doi.org/10.18632/oncotarget.11658

Wettersten, H. I., Hakimi, A. A., Morin, D., Bianchi, C., Johnstone, M. E., Donohoe, D. R., et al. (2015). Grade-dependent metabolic reprogramming in kidney cancer revealed by combined proteomics and metabolomics analysis. Cancer Research, 75(12), 25412552. https://doi.org/10.1158/0008-5472.CAN-14-1703

Wishart, D. S., Tzur, D., Knox, C., Eisner, R., Guo, A. C., Young, N., et al. (2007). HMDB: The human metabolome database. Nucleic Acids Research, 35(Database), 521-526. https://doi.org/10.1093/ nar/gk1923

Publisher's Note Springer Nature remains neutral with regard to jurisdictional claims in published maps and institutional affiliations. 